\title{
COMMONSENSE METAPHYSICS AND LEXICAL SEMANTICS
}

\author{
Jerry R. Hobbs, William Croft, Todd Davies, \\ Douglas Edwards, and Kenneth Laws \\ Artificial Intelligence Center \\ SRI International
}

\section{Introduction}

In the TACITUS project for using commonsense knowtedge in the understanding of texts about mechanical devices and their failures, we have been developing various commonsense theories that are needed to mediate between the way we talk about the behavior of such devices and causal models of their operation. Of central importance in this effort is the axiomatization of what might be called "commonsense metaphysics". This includes a number of areas that figure in virtually every domain of discourse, such as scalar notions, granularity, time, opace, material, physical objects, causality, functionality, force, and shape. Our approach to lexical semantics is then to construct core theories of each of these areas, and then to define, or at least characterize, a large number of lexical items in terms provided by the core theories. In the TACITUS aystem, processes for solving pragmatics problems posed by a text will use the knowledge base consisting of these theories in conjunction with the logical forms of the centences in the text to produce an interpretation. In this paper we do not stress these interpretation proceses; this is anotber, important aspect of the TACITUS project, and it will be described in subsequent papers.

This work represents a convergence of reweard in lexical semantics in linguistics and effort in $\mathrm{AI}$ to encode commonsense knowledge. Lexical semantjcints over the years bave developed formalisms of increasing adequacy for encoding word meaning, progressing from simple wets of fastures (Katz and Fodor, 1963) to notatione for prodicatoargument structure (Lakofi, 1972; Miller and JohneonLaird, 1976), but the early attempto atill limited accens to world knowledge and assumed only very restricted corts of processing. Workers in computational linguistics introduced inference (Rieger, 1974; Schank, 1975) and other complex cognitive processes (Herskovits, 1982) into our understanding of the role of word meaning. Recently, linguists bave given greater attention to the cognitive processes that would operate on their represeatations (e.g., Talmy, 1983; Croft, 1986). Independently, is Al an effort arose to encode large amounts of commonsease knowl- odge (Hayes, 1979; Hobbs and Moore, 1985; Hobbs et al 1985). The reseanch reported bere represents a convergence of these various developments. By developing core theories of several fundamental phenomena and defining lexical items witbin these theories, using the full power of predicate calculus, we are able to cope with complexities of word meaning that have hitberto escaped lexical cemanticists, within a framework that gives full scope to the planning and reasoning processes that manipulate representations of word meaning.

In constructing the core theories we are attempting to adhere to several methodological principles.

1. One should aim for characterization of concepts. ratber than definition. One cannot generally expect to find necessary and sufficient conditions for 2 concept. The most we can hope for is to find a number of necessary conditions and a number of sufficient conditions. This amounts to saying that a great many predicales are primitive, but primitives that are highly interrelated with the rest of the knowledge base.

2. One should determine the minimal structure necesary for a concept to make sense. In efforts to axiomatize come area, there are two positions one may take, exemplified by eet theory and by group theory. In axiomatizing wet theory, ope attempts to capture exactly some concept one has strong intuitions about. If the axjomatization turwe out to have unexpected models, this exposes $2 n$ inadequacy. In group theory, by contrast, one characterizes an abstract clase of structures. If there turn out to be unexpected models, this is a serendipitous discovery of a new phenomenon that we can reason about using an old theory. The pervasive character of metaphor in aatural language discourse shows that our commonsense theories of the world ought to be much more like group theory than set theory. By seeking minimal structures in axiomatizing concepts, we optimize the possibilities of using the theories in metaphorical and analogical contexts. This principle is illustrated below in the section on regions. One consequence of this principle is that our approach will seem more syntactic than emantic. We have concentrated more on 
specifying axjoms than on constructing models. Our view is that the chief role of models in our efiort is for proving the consistency and independence of sets of axioms, and for showing their adequacy. As an example of the last point, many of the spatial and temporal theories we construct are intended at least to have Euclidean space or the real numbers as one model, and a subclaes of graph-theoretical structures as other models.

3. A balance must be struck between attempting to cover all cases and aiming only for the prototypical cases. In general, we have tried to cover as many cases a possible with an elegant axiomatization, in line with the two previous principles, but where the formalization begins to look baroque, we assume that higher processes will suspend some inferences in the marginal cases. We assume that inferences will be drawn in a controlled fashion. Thus, every outré, highly context-dependent counterexample need not be accounted for, and to a certain extent, definition can be geared specifically for a prototype.

4. Where competing ontologies suggest themsetves in a domain, one sbould attempt to construct a theory that accommodates both. Ratber than commit oneself to adopting one set of primitives rather than another, one should show how each of of primitives can be characterized in terms of the other. Generally, each of the ontologies is useful for different purposes, and it is convenient to be able to appeal to both. Our treatment of time illustrates this.

5. The theories one constructs should be richer in axioms than in theorems. In mathematics, one expects to state half a dozen axioms and prove dozens of theorems from them. In encoding commonsense knowledge it ceems to be just the opposite. The theorems we reek to prove on the basis of these axioms are theorems about specific situation which are to be interpreted, in particular, theorems about a text that the system is attempting to understand.

6. One should avoid falling into "black holes". There are a lew "mysterious" concepts which crop op repestedly in the formalization of commonsense metaphysica. Amons these are "relevant" (that is, relevant to the task at hand) and "normative" (or conforming to some norm or pattern). To insist upon giving a satisfactory analysis of theae before using them in analyzing other concepts is to crom the event horizon that separates lexical semantics from philosophy. On the other band, our experience suggests that to avoid their use entirely is crippling; the lexical semantic of a wide variety of otber terms depends upon them. Inatead, we have decided to leave them minimally analyzed for the moment and use them without acruple in the analyais of otber commonsense concepts. This approach will allow us to accumulate many examples of the use of these mysterious concepts, and in the end, contribute to their succeseful analysis. The use of these concepts appears below in the discussions of the words "immediately", "sample", and "operate".
We chose as an initial target problem to encode the commonsense knowledge that underlies the concept of "wear", $a$ in a part of a device wearing out. Our aim was to define "wear" is termu of predicates characterized elsewbere in the knowledge base and to infer consequences of wear. For comething to wear, we decided, is for it to lose imperceptible bits of material from its surface due to abrasive action over time. One goal, which we have not yet achieved, is to be able to prove as a theorem that since the shape of a part of a mechanical device is often functional and since loss of material can result in a change of shape, wear of a part of - device can result in the failure of the device as a whole. In addition, as we have proceded, we have characterized a number of words found in a set of target texts, as it has become possible.

We are encoding the knowledge as axioms in what is for the moet part a first-order logic, described in Hobbs (1985a), although quantification over predicates is sometimes convenient. In the formalism there is a nominalization operator * * for reifying events and conditions, 28 expreseed in the following axiom schema:

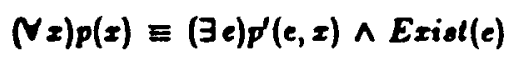

That is, $p$ is true of $x$ if and only if there is a condition $e$ of $p$ being true of $x$ and $e$ exists in the real world.

In our implementation so far, we have been proving simple theorems from our axioms using the CG5 theoremprover developed by Mark Stickel (1982), but we are only now beginning to use the knowledge base in text processing.

\section{Requirements on Arguments of Predicates}

There is a notational convention used below that deserves come explanation. It has frequently been noted that relational words in natural language can take only certain types of words a their arguments. These are usually described as selectional constraints. The same is true of predicates in our knowledge base. They are expressed below by rules of the form

$$
p(x, y): r(x, y)
$$

This means that for $p$ even to make sense applied to $x$ and $y$, it muat be the case that $r$ is true of $x$ and $y$. The logical import of this rule is that wherever there is an axiom of the form

$$
(\forall x, y) p(x, y) \supset q(x, y)
$$

this is really to be read as

$$
(\forall x, y) p(x, y) \wedge r(x, y) \supset q(x, y)
$$


The checking of selectional constraints, therefore, falls out as a by-product of other logical operations: the constraint $r(x, y)$ must be verified if anything else is to be proven from $p(x, y)$.

The simplest example of such an $r(x, y)$ is a conjunction of sort constraints $r_{1}(x) \wedge r_{2}(y)$. Our approach is a generalization of this, because much more complex requirements can be placed on the arguments. Consider, for example, the verb "range". If I ranges from $y$ to $z$, there must be $a$ scale o that includes $y$ and $z$, and $z$ must be $a$ set of entities that are located at various places on the scale. This can be represented as follows:

$$
\begin{aligned}
& \operatorname{range}(x, y, z):(\exists \bullet) \text { scale }(\bullet) \wedge y \in \bullet \\
& \wedge z \in \bullet \wedge \operatorname{vet}(z) \\
& \wedge(\forall u)[u \in \Sigma \supset(\exists v) v \in \bullet \wedge \in(z, v)]
\end{aligned}
$$

\section{The Knowledge Base}

\subsection{Sets and Granularity}

At the foundation of the knowledge base is an axiomatization of set theory. It follows the standand Zermelo-Frankel approach, except that there is no Axiom of Infinity.

Since so many concepts used in discourse are graindependent, a theory of granularity in also fuadamental (see Hobbs 1985b). A grain is defined in terms of an indistinguishability relation, which is reflexive and symmetric, but not necessarily transitive. One grain can be a refinement of another with the obvious definition. The most refined grain is the identity grain, i.e., the one in which every two distinct elements are distinguishable. One possible relar tionship between two grains, one of which is a relnement of the other, is what we call an "Archimedean relation", after the Archimedean property of real numbers. Intvitively, if enough events occur that are imperceptible at the coarser grain $g_{2}$ but perceptible at the finer grain $g_{h}$, then the aggregate will eventually be perceptible at the coarnt grain. This is an important property in phenomeas subject to the Heap Paradox. Wear, for instance, eventually has significant consequences.

\subsection{Scales}

A great many of the most common words in Englinh have acales as their subject matter. This includes many preponitions, the most common adverbe, comparatives, and many abatract verbo. When spatial vocabulary is uced metaphor ically, it is generally the scalar aspect of space that carrie over to the target domain. A scale is defined as a set of elements, together with a partial ordering and a granularity (or an indistinguishability relation). The partial or dering and the indistinguisbability relation are consistent with each other:

$$
(\forall x, y, z) x<y \wedge y \sim z \supset x<z \vee x \sim z
$$

It is useful to have an adjacency relation between points on acale, and there are a number of ways we could introduce it. We could simply take it to be primitive; in a scale having a distance function, we could define two points to be adjacent when the distance between them is less than come $\epsilon$; finally, we could defioe adjacency in terms of the grain-size:

$$
N z, y, \operatorname{\theta odj}(x, y, \theta) \equiv
$$

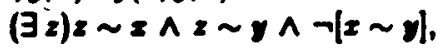

Two important possible properties of scales are connectodneas and denseness. We cas say that two elements of a acale are connected by a chain of adj relations:

$$
\begin{aligned}
& N x, y, \bullet) \text { connected }(x, y, \bullet) \equiv \\
& \quad \text { adj }(x, y, \bullet) \vee \\
& (\exists z) \text { adj }(x, z, \bullet) \wedge \text { connected }(z, y, \theta)
\end{aligned}
$$

A ecalo in connected (oconnecfed) if all pairs of elements ar connected. A scale is dense if between any two points there in a thind point, nntil the two points are so close together that the grain-size won't let us tell what the situation in. Cranking up the magaification could well resolve the continuous apace into a discrete set, as objects into atoms.

$$
\begin{aligned}
& \text { No)dence(o) } \equiv \\
& (\forall x, y,<) x \in \Delta \wedge y \in \Delta \wedge \operatorname{order}(<, \Delta) \wedge x<y \\
& \supset(\exists z)(x<z \wedge z<y) \\
& \vee(\exists z)(z \sim z \wedge z \sim y)
\end{aligned}
$$

This captures the commonsense notion of continuity.

A eubecale of a scale bas as its elements a subset of the elements of the ecale and has as its partial ordering and its srain the partial ordering and the grain of the scale.

$$
\begin{aligned}
& \left(V_{1},<, \sim \operatorname{lorder}\left(<_{1} \theta_{1}\right) \wedge \operatorname{grain}\left(\sim, \theta_{1}\right)\right. \\
& \supset \text { (Vos)loubecale }\left(\theta_{2}, \theta_{1}\right) \\
& \left.\equiv \text { oubset }\left(\bullet_{2}, \bullet_{1}\right) \wedge \operatorname{order}\left(<, \theta_{2}\right) \wedge \operatorname{grain}\left(\sim, \bullet_{2}\right)\right]
\end{aligned}
$$

An interval can be defined as a connected subscale:

$$
\text { Ni)interval(i) } \equiv(\exists \text { o)ecale(o) }
$$

$$
\wedge \text { subseale }(i, \bullet) \wedge \text { sconnected }(i)
$$

The relations between time intervals that Allen and Kauts (1985) have defined can be defined in a straightforward manner in the approach presented bere, applied to intervale in renerd.

A coscept closely related to scales is that of a "cycle". This is a ryotem which has a natural ordering locally but contains s loop globally. Examples include the color wheel, clock timen, and seographical locations ordered by "east of". Wo have exiomatized cycles ini terms of a ternary batween relation, whose axioms parallel the axioms for a partial ordering.

The figure-ground relationship is of fundamental importance in language. We eacode this with the primitive predicate at. The minimal structure that seems to be necessary for something to be a ground is that of a scale; bence, this is a selectional constraint on the arguments of at. 


$$
\text { al }(x, y):(\exists \bullet) y \in \bullet \wedge \text { ocale( }(\bullet)
$$

At this point, we are already in a position to define some fairly complex words. As an illustration, we give the example of "range" as in " $x$ rages from $y$ to $z^{n}$ :

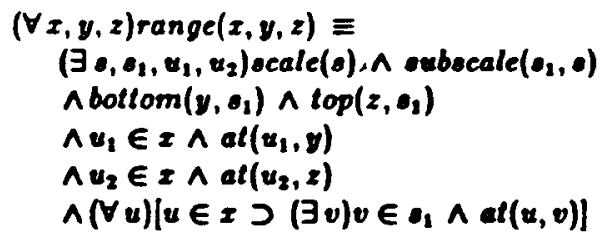

A very important scale is the linearly ondered scale of numbers. We do not plan to reason axiomatically about numbers, but it is useful in natural language processing to have encoded a few facts about numbers. For example, a set bas a cardinality which is an element of the number scale.

Verticality is a concept that would be most properly analyzed in the section on space, but it is a property that many other scales have acquired metaphorically, for whatever reason. The number scale is one of these. Even in the absence of an analysis of verticality, it is a useful property to have as a primitive in lexical semantics.

The word "high" is a vague term that asserts an entity is in the upper region of some scale. It requires that the ecale be a verlical one, such as the number scale. The verticality requirement distinguishes "bigh" from the more general term "very"; we can say "very bard" but not "bighly bard". The phrase "bighly planar" sounds all right because the high register of "planar" suggests a quantifiable, scientific accuracy, whereas the low register of "fat" makes "highly flat" sound much worse.

The test of any definition is whether it allows one to draw the appropriate inferences. In our target texts, the phrace "higb usage" occurs. Usage is a set of using events, and the verticality requirement on "high" forces us to coerce the phrase into "a high or large number of using events". Combining this with an axiom that says that the use of a mochanical device involves the likelihood of abrasive events, as defined below, and with the definition of "wear" in term: of abrasive events, we should be able to conclude the likelibood of wear.

\subsection{Time: Two Ontologies}

There are two possible ontologies for time. In the first, the one most acceptable to the mathematically minded, there is a time line, which is a scale having some topological structure. We can stipulate the time line to be linearly ordered (altbough it is not in approaches that build ignorance of relative times into the representation of time (e.g., Hobbs, 1974) nor in approaches using branching futures (e.8., McDermott, 1985)), and we can stipulate it to be dense (although it is not in the situation calculus). We take before to be the ordering on the time line:

$$
\begin{aligned}
& \left(\forall t_{1}, t_{2}\right) \text { be fore }\left(t_{1}, t_{2}\right) \equiv \\
& (\exists T,<) T \text { Time-line }(T) \wedge \operatorname{order}(<, T) \\
& \wedge t_{1} \in T \wedge t_{2} \in T \wedge t_{1}<t_{2}
\end{aligned}
$$

We allow both instants and intervals of time. Most events occur at some instant or during some interval. In this epproach, nearly every predicate takes a time argument.

In the second ontology, the one that seems to be more deeply rooted in language, the world consists of a large number of more or less independent processes, or histories, or sequences of events. There is a primitive relation change between conditions. Thus,

$$
\text { change }\left(e_{1}, e_{2}\right) \wedge \boldsymbol{p}\left(e_{1}, x\right) \wedge \boldsymbol{q}\left(e_{2}, x\right)
$$

ays that there is a change from the condition $e_{1}$ of $p$ being true of 2 to the condition $e_{2}$ of $q$ being true of $x$.

The time line in this ontology is then an artificial conotruct, a regular sequence of imagined abstract eventsthink of them as ticks of a clock in the National Bureau of Standards-to which other events can be related. The change ontology reems to correspond to the way we experience the world. We recognize relations of causality, change of state, and copresence among events and conditions. When events are not related in these ways, judgments of relative time must be mediated by copresence relations between the events and events on a clock and change of atate relations on the clock.

The predicate change possesses a limited transitivity. There has been a change from Reagan being an actor to Reagan being President, even though he was governor in between. But we probably do not want to say there bas been a change from Reagan being an actor to Margaret Thatcher being Prime Minister, even though the second comes after the first.

We can way that times, viewed in this ontology as events, sways have s change relation between them.

$$
\left.N t_{1}, t_{2}\right) \text { be } \operatorname{fore}\left(t_{1}, t_{2}\right) \supset \text { change }\left(t_{1}, t_{2}\right)
$$

The predicate change is related to before by the axiom

$$
\begin{aligned}
& \left.N e_{1}, e_{2}\right) \text { change }\left(e_{1}, e_{2}\right) \supset \\
& \left(\exists t_{1}, t_{2}\right) \text { at }\left(e_{1}, t_{1}\right) \\
& \wedge \text { at }\left(e_{2}, t_{2}\right) \wedge \operatorname{tefore}\left(t_{1}, t_{2}\right)
\end{aligned}
$$

This does not allow us to derive change of state from temporal euccesaion. For this, we need axioms of the form

$$
\begin{aligned}
& \left.N e_{1}, e_{3}, t_{1}, t_{2}, x\right) p^{\prime}\left(e_{1}, x\right) \wedge a t\left(e_{1}, t_{1}\right) \\
& \wedge q^{\prime}\left(e_{2}, x\right) \wedge a t\left(e_{2}, t_{2}\right) \wedge \text { before }\left(t_{1}, t_{2}\right) \\
& \supset \text { change }\left(e_{1}, e_{2}\right)
\end{aligned}
$$

That is, if $I$ is $p$ at time $l_{1}$ and $q$ at a later time $t_{2}$, then there has been a change of state from one to the other. Time arguments in predications can be viewed as abbreviations:

$$
\forall x, t) p(x, t) \equiv(\exists e) p^{\prime}(e, x) \wedge \operatorname{at}(e, t)
$$


The word "move", or the predicate move, (as in "I moves from $y$ to $z^{\prime \prime}$ ) can then be defined equivaleatly in terms of change

$$
\begin{aligned}
& (\forall x, y, z) \text { move }(x, y, z) \equiv \\
& \left(\exists e_{1}, e_{2}\right) \text { change }\left(e_{1}, e_{z}\right) \\
& \wedge a t^{\prime}\left(e_{1}, x, y\right) \wedge a f^{\prime}\left(e_{z}, x, z\right)
\end{aligned}
$$

or in terms of the time line

$$
\begin{aligned}
& (\forall x, y, z) \text { move }(x, y, z) \equiv \\
& \left(\exists t_{1}, t_{2}\right) \text { at }\left(x, y, t_{1}\right) \wedge \text { at }\left(x, z, t_{2}\right) \wedge \text { before }\left(t_{1}, t_{2}\right)
\end{aligned}
$$

In English and apparently all other natural languages, both ontologies are represented in the lexicon. The time line ontology is found in clock and calendar terms, tense systems of verbs, and in the deictic temporal locatives such as "yesterday", "today", "tomorrow", "lest night", and 10 on. The change ontology is exhibited in most verbs, and in temporal clausal connectives. The universal presence of both classes of lexical items and grammatical markers in natural languages requires a theory which can accommodate both ontologies, illustrating the importance of metbodological principle 4.

Among temporal connectives, the word "while" presents interesting problems. In " $c_{1}$ while $e_{2}$ ", $e_{2}$ must be an event occurring over a time interval; $e_{1}$ must be an event and may occur eitber at a point or over an interval. One's fint guess is that the point or interval for $e_{1}$ must be included in the interval for $e_{2}$. However, there are cases, such w

It rained while 1 was in Philadelphia.

or

The electricity should be of while the switch i being repaired.

which suggest the reading " $e_{2}$ is included in $e_{1}$ ". We came to the conclusion that one can infer no more than that $e_{1}$ and $e_{2}$ overlap, and any tighter constraints result from implicatures from background knowledge.

The word "immediately" also presents a number of problems. It requires its argument $e$ to be an ordering relation between two entities $x$ and $y$ on some scale 0 .

$$
\text { immediate(e): }(\exists x, y, s) \text { Ieso-lhan' }(e, z, y, e)
$$

It is not clear what the constraints on the scale are. Temporal and spatial scales are okay, 25 in "immediately after the alarm" and "immediately to the left", but the aize acale isn't:

- John is immediately larger than Bill.

Etymologically, it means that there are no intermediate entities between $x$ and $y$ on 8 . Thus,

$$
\begin{aligned}
& \forall e, x, y, s) \text { immediate }(e) \wedge \text { less-than' }(e, x, y, \theta) \\
& \supset \neg(\exists z) \text { less-than }(x, z, \theta) \wedge \text { less-than }(z, y, \theta)
\end{aligned}
$$

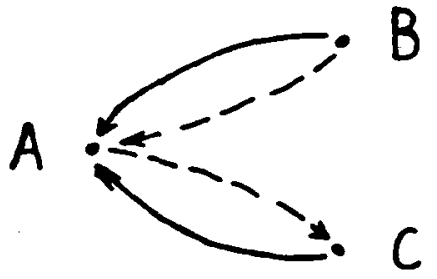

Figure 1: The simplest space.

Bowever, this will only work if we restrict $z$ to be a relevant entity. For example, in the sentence

We disengaged the compressor immediately after the alarm.

the implication is that no event that could damage the compressor occurred between the alarm and the disengagement, wince the text is about equipment failure.

\subsection{Spaces and Dimension: The Minimal Structure}

The notion of dimension has been made precise in linear alrebra. Since the concept of a region is used metaphorically $\boldsymbol{a}$ well $\boldsymbol{a}$ in the spatial sense, however, we were concerned to determine the minimal structure that a system requires for it to make sense to call it a space of more than one dimention. For a two-dimensional space, there must be a cale, or partial ordering, for each dimension. Moreover. the two cealea must be independent, in that the order of elements on one acale can not be determined from their onder on the other. Formally,

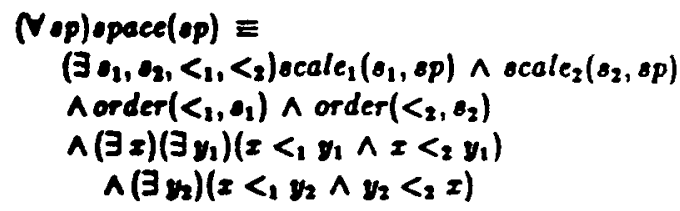

Note that this does not allow $<_{2}$ to be simply the reverse of $<_{1}$. An uncurprising consequence of this definition is that the minimal example of a two-dimensional space consists of three points (three points determine a plane), e.g., the pointe $A, B$, and $C$, where

$$
A<_{1} B, A<_{1} C, C<_{2} A, A<_{2} B \text {. }
$$

This is lllustrated is Figure 1.

The dimensional scales are apparently found in all natural languages in relevant domains. The familiar three dimensional space of common sense is defined by the three scale pairs "up-down", "front-back", and "left-right"; the two-dimensional plane of the commonsense conception of the earth's surface is represented by the two scale pairs "nortb-soutb" and "east-west". 
The simplest, although not the only, way to define adjacency in the space is as adjacency on both scales:

$$
\begin{aligned}
& (\forall x, y, o p) \operatorname{adj}(x, y, s p) \equiv \\
& \left(\exists s_{1}, s_{2}\right) \text { ocale } e_{1}\left(o_{1}, s p\right) \wedge \text { ocale }\left(s_{2}\left(s_{2}, s p\right)\right. \\
& \wedge \operatorname{adj}\left(x, y, o_{1}\right) \wedge \operatorname{adj}\left(x, y, o_{2}\right)
\end{aligned}
$$

A region is a subset of a space. The aurface and interior of 2 region can be defined in terms of adjacency, in a manner paralleling the definition of a boundary in point-set topology. In the following, $s$ is the boundary or surface of a twoor three-dimensional region $r$ embedded in a space op.

$(\forall s, r)$ surface $(s, r, s p) \equiv$

$(\forall x) x \in r \supset[x \in: \equiv$

$(E y)(y \in \operatorname{sp} \wedge \neg(y \in r) \wedge \operatorname{adj}(x, y, o p))]$

Finally, we can define the notion of "contact" in terms of points in different regions being adjacent.

$$
\begin{aligned}
& \left(\forall r_{1}, r_{2}, o p\right) \text { contact }\left(r_{1}, r_{2}, o p\right) \equiv \\
& \quad \operatorname{disjoint}\left(r_{1}, r_{2}\right) \wedge \\
& (E x, y)\left(x \in r_{1} \wedge y \in r_{2} \wedge \operatorname{adj}(x, y, \Delta p)\right)
\end{aligned}
$$

By picking the scales and defining adjacency right, we can talk about points of contact between communicational Detworks, systems of knowledge, and other metaphorical domains. By picking the scales to be the real line and defining adjacency in terms of $\epsilon$-neighborhoods, we get Euclidean space and can talk about contact between physical objects.

\subsection{Material}

Physical objects and materials must be distinguished, just as they are apparently distinguished in every natural language, by means of the count noun - mass noun distinction. A physical object is not a bit of material, but rather is comprised of a bit of material at any given time. Thus, rivers and human bodies are physical objects, even though their material constitution changes over time. This distinetion also allows us to talk about an object losing material through wear and still being the same object.

We will say that an entity $b$ is a bit of material by means of the expression material $(b)$ : Bite of material are characterized by both extension and cohesion. The primitive predication occupies $(b, r, t)$ encodes extension, saying that - bit of material 6 occupies a region $r$ at time $t$. The topoloby of a bit of material is then parasitic on the topology of the region it occupies. A part $b_{1}$ of a bit of material $b$ is a bit of material whose occupied region is always a subregion of the region occupied by b. Point-like particles (particle) are defined in terms of points in the occupied region, disjoint bits (diajointbil) in terms of disjointness of regions, and contact between bits in terms of contact between their regions. We can then state as follows the Principle of NonJoint-Occupancy that two bits of material cannot occupy the same place at the same time:

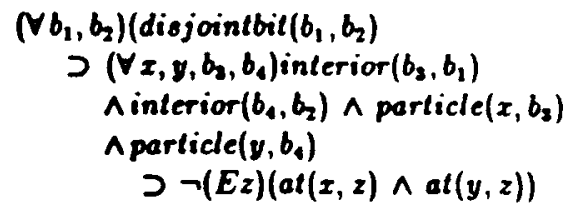

At some future point in our work, this may emerge as a consequence of a richer theory of cohesion and force

The cohesion of materials is also a primitive property; for we must distinguish between a bump on the surface of an object and a chip merely lying on the surface. Cobesion depends on a primitive relation bond between particles of material, paralleling the role of adj in regions. The relation attached is defined as the transitive closure of bond. A topology of cohesion is built up in a manner analogous to the topology of regions. In addition, we have encoded the relation that bond bears to motion, i.e. that bonded bito remain adjacent and that one moves when the other does, and the relation of bond to force, i.e. that there is a characteristic force that breaks a bond in a given material.

Different materials react in different ways to forces of various strengths. Materials subjected to force exhibit or fail to exhibit several invariance properties, proposed by Hager (1985). If the material is shape-invariant with respect to a particular force, its shape remains the same. If it is topologically invariant, particles that are adjacent remain adjacent. Shape invariance implies topological invariance. Subject to forces of a certain strength or degree $d_{1}$, a material ceases being shape-invariant. At a force of etrength $d_{2} \geq d_{1}$, it ceases being topologically invariant, and at a force of strength $d_{3} \geq d_{2}$, it simply breaks. Metals exhibit the full range of possibilities, that in, $0<d_{1}<d_{2}<d_{2}<\infty$. For forces of strength $d<d_{1}$, the material is "bard"; for forces of strength $d$ where $d_{1}<d<d_{2}$, it is "flexible"; for forces of strength $d$ where $d_{2}<d<d_{3}$, it is "malleable". Words such as "ductile" and "elastic" can be defined in terms of this voeabulary, logether with predicates about the geometry of the bit of material. Words such as "brittle" $\left(d_{1}=d_{2}=d_{2}\right)$ and "Euid" $\left(d_{2}=0, d_{2}=\infty\right)$ can also be defined in these terms. While we should not expect to be able to definc various material terms, like "metal" and "ceramic", we can certainly characterize many of their properties with this vocabulary.

Because of its invariance properties, material interacts with containment and motion. The word "clog" illustrates this. The predicate clog is a three-place relation: $x$ clogs $y$ aginst the low of $z$. It is the obstruction by $z$ of $z$ 's motion through $y$, but with the selectional restriction that s must be something that can flow, such as a liquid, gas, or powder. If a rope is passing through a bole in 2 board, and a knot in the rope prevents it from going tbrough, we do not say that the bole is clogged. On the other hand, there do not seem to be any selectional constraints on $x$. In particular, I can be identical with z: glue, sand, or molasses can clog a passageway against its own flow. We 
can speak of clogging where the obstruction of tow in not complete, but it must be thought of as "nearly" complete.

\subsection{Other Domains}

\subsubsection{Cancal Connection,}

Attachment within materials is one variety of causal connection. In general, if two entities $x$ and $y$ are causally connected with respect to some behavior $p$ of $x$, then whenever $p$ happens to $x$, there is some corresponding behavior $q$ that happens to $y$. In the case of attachment, $p$ and $q$ are botb move. A particularly common variety of causal connection between two entities is one mediated by the motion of a third entity from one to the other. (This might be called a "vector boson" connection.) Photons mediating the connection between the oun and our eyes, nin drops connecting a state of the clouds with the wetness of our skin and clothes, a virus being transmitted from one person to another, and utterances passing between people are all examples of sucb causal connections. Barriers, openings, and penetration are all with respect to paths of causal connection.

\subsubsection{Foree}

The concept of "force" is axiomatized, in a way consistent witb Talmy's treatment (1985), in terms of the predications force $\left(a, b, d_{1}\right)$ and resiat $\left(b, a, d_{2}\right)-a$ forces against $b$ with strength $d_{1}$ and $b$ resists $a$ 's action with strength $d_{2}$. We can infer motion from facts about relative strengtb. This treatment can also be specialized to Newtonian force, where we have not merely movement, but acceleration. In addition, in spaces in wbich orientation is defined, forces can have an orjentation, and a version of the Parallelogram of Forces Law can be encoded. Finally, force interacts with shape in ways characterized by words like "stretch", "compress", "bend", "twist", and "shear".

\subsubsection{Syatems and Functionality}

An important concept is the notion of a "system", which is a set of entities, a set of their properties, and a set of relations among them. A common kind of oystem is one in which the entities are events and conditions and the relations are causal and enabling relations. A mechanical device can be described as such a system-in a sense, in terms of the plan it executes in its operation. The function of various parts and of conditions of those parts is then the role they play in this system, or plan.

The intransitive sense of "operate", 20 in

The diesel was operating.

involves systems and functionality. If an entity $z$ operates, then there must be a larger system 8 of which $z$ is a part. The entity $x$ itself is 2 system with parts. These parts undergo normative state changes, thereby causing I to undergo normative atate changes, thereby causing $x$ to produce an effect with a normative function in the larger cystem. . The concept of "normative" is discussed below.

\subsubsection{Bhape}

We have been approaching the problem of characterizing shape from a number of different angles. The classical treatment of shape is via the notion of "similarity" in Euclidean geometry, and in Bilbert's formal reconstruction of Euclidean geometry (Hilbert, 1902) the key primitive concept seems to be that of "congruent angles". Therefore, we first sought to develop a theory of "orientation". The chape of an object can then be characterized in terms of changes in orientation of a tangeni as one moves about on the eurface of the object, as is done in vision research (e.g., Zahn and Roskies, 1972). In all of this, since "shape" can be used loosely and metaphorically, one question we are aking is whether some minimal, abstract structure can be found in which the notion of "shape" makes sense. Conoider, for instance, a graph in which one scale is discrete, or even unordered. Accordingly, we have been examiniog a number of examples, 2sking when it seems right i.o say two atructures have different shapes.

We bave also examined the interactions of shape and functionality (cf. Davis, 1984). What seems to be crucial is bow the shape of an obstacle constrains the motion of a substance or of an object of a particular sbape (cf. Shobam, 1985). Thus, a fundel concentrates the flow of a liquid, and similarly, a wedge concentrates force. A box pushed against a ridge in the foor will topple, and a wheel is a limiting case of continuous toppling.

\subsection{Hitting, Abrasion, Wear, and Re- lated Concepts}

For $x$ to hit $y$ is for $x$ to move into contact with $y$ with some force.

The basic ecenario for an abrasive event is that there is on impinging bit of material $m$ which hits an object $o$ and by doing 20 removes a pointlike bit of material $b_{0}$ from the anriace of o:

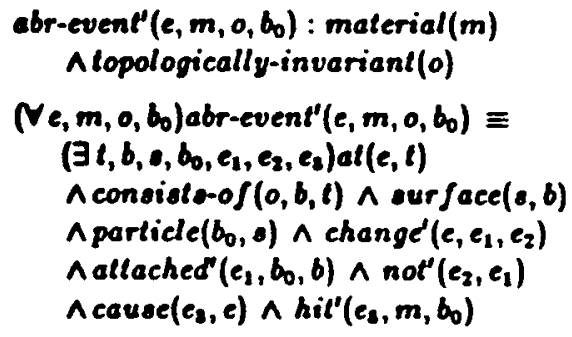

After the abrasive event, the pointlike bit $b_{0}$ is no longer a part of the object 0 : 


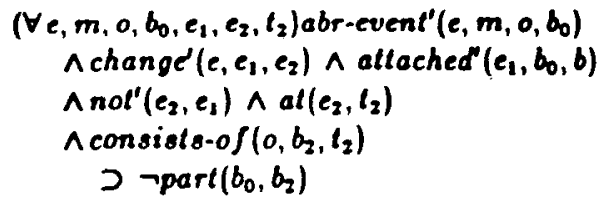

It is necessary to state this explicitly since objects and bits of material can be discontinuous.

An abrasion is a large number of abrasive events widely distributed through some nonpointlike region on the surface of an object:

$$
\begin{aligned}
& \text { ( } \forall c, m, o) a b r a d e(e, m, o) \equiv \\
& (\exists b s)\left\|\left(\forall e_{1}\right)\right\| e_{1} \in e \supset \\
& \left.\left(\exists b_{0}\right) b_{0} \in b_{b} \wedge \text { abr-event }\left(e_{1}, m, 0, b_{0}\right)\right] \\
& \wedge(\forall b, s, t) \mid a t(c, t) \\
& \text { A consiats-of }(0, b, t) \wedge \text { eur } f a c e(s, b) \\
& \supset \text { ( } \exists \text { rloubregion }(r, a) \\
& \wedge \text { widely-distributed }(b e, r)]]
\end{aligned}
$$

Wear can occur by means of a large collection of abrasive events distributed over time as well as space ( 20 that there may be no time at which enough abrasive events occur to count as an abrasion). Thus, the link betwoen wear and abrasion is via the common notion of abrasive events, not via a definition of wear in terms of abrasion.

$$
\begin{aligned}
& (\forall e, m, o) \text { wear' }(e, x, o) \equiv \\
& (\exists b s)\left(\forall e_{1}\right)\left(e_{1} \in e \supset\right. \\
& \left.\left.\left(\exists b_{0}\right) b_{0} \in b_{s}\right) \wedge \text { abr-event' }\left(e_{1}, m, 0, b_{0}\right)\right] \\
& \wedge(\exists i) \mid \text { inlerval }(i) \wedge \text { widely-diolributed }(e, i)]
\end{aligned}
$$

The concept "widely distributed" concerns systems. If $x$ is distributed in $y$, then $y$ is a oystem and $x$ is a set of entities which are located at components of $y$. For the distribution to be wide, most of the elements of a partition of $y$ determined independently of the dintribution must contain components which have elemente of 2 at them.

The word "wear" is one of a large class of other events involving cumulative, gradual loss of material - events described by words like "chip", "corrode", "file", "erode", "rub", "sand", "grind", "weather", "ruat", "tarnish", "eat sway", "rot", and "decay". All of these lexieal items can now be defined as variations on the definition of "wear", since we have built up the axiomatizations undertying "wear". We are now in a position to characterize the edtire class. We will illustrate this by defining two different types of variants of "wear" - "chip" and "corrode".

"Chip" differs from "wear" in three ways: the bit of material removed in one abrasive event is larger (it need not be point-like), it need not happen because of a material bitting against the object, and "chip" does not require (thougb it does permit) a large collection of such events: one can say that some object is chipped if there is only one chip in it. Thus, we slightly alter the definition of abr-event to accommodate these changes:

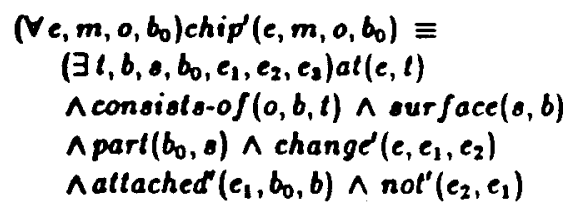

"Corrode" differs from "wear" in that the bit of material is chemically transformed as well as being detached by the contact event; in fact, in some way the chemical transformation causes the detachment. This can be captured by adding a condition to the abrasive event which renders it a (single) corrode event:

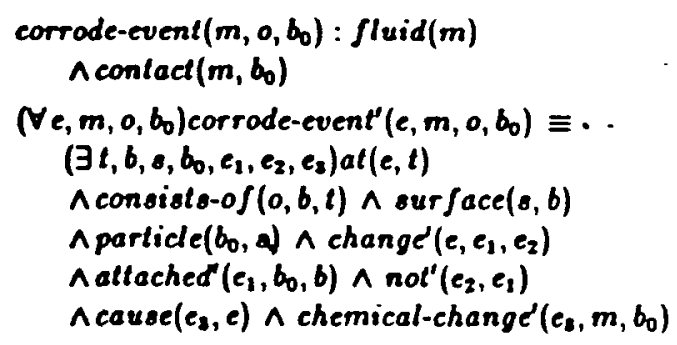

"Corrode" itself may be defined in a parallel fashion to "wear", substituting corrode-event for abr-event.

All of this suggests the generalization that abrasive events, chipping and corrode events all detach the bit in question, and that we may describe all of these as detaching events. We can then generalize the above axiom about abrasive events resulting in loss of material to the following axiom about detaching:

$$
\begin{aligned}
& \text { (Ve, } \left.m, 0, b_{0}, b_{2}, c_{1}, c_{2}, t_{2}\right) \operatorname{detach}^{\prime}\left(e, m, 0, b_{0}\right) \\
& \wedge \text { change' }\left(e, e_{1}, e_{2}\right) \wedge \text { attached }\left(e_{1}, b_{0}, b\right) \\
& \wedge \operatorname{not}^{\prime}\left(e_{2}, e_{1}\right) \wedge a t\left(e_{2}, t_{2}\right) \\
& \wedge \text { consists-of }\left(0, b_{2}, t_{2}\right) \\
& \supset \neg\left(\operatorname{part}\left(b_{0}, b_{2}\right)\right)
\end{aligned}
$$

\section{Relevance and the Normative}

Many of the concepts we are investigating have driven us inexorably to the problems of what is meant by "relevant" and by "normative". We do not pretend to have solved these problems. But for each of these concepts we do have the beginnings of an account that can play a role in analyais, if not yet in implementation.

Our view of relevance, briefly stated, is that something is relevant to some goal if it is a part of a plan to achieve that goal. (A formal treatment of a similar view is given in Davies and Russell, 1986.) We can illustrate this with an example involving the word "sample". If a bit of material $z$ is a sample of another bit of material $y$, then $z$ is a part of $y$, and moreover, there are relevant properties $p$ and $q$ such that it is believed that if $p$ is true of $x$ then $q$ is true of $y$. That is, looking at the properties of the sample telis us something important about the properties of the whole. Frequently, $p$ and $q$ are the same property. In our target texts, the following sentence occurs: 
We retained an oil sample for future inspection.

The oil in the sample is a part of the total lube oil in the lube oil system, and it is believed that a property of the sample, such as "contaminated with metal particles", will be true of all of the lube oil as well, and that this will give information about possible wear on the bearings. It is therefore relevant to the goa of maintaining the machinery in good working order.

We have arrived at the following provisional account of what it means to be "normative". For an entity to exhibit a normative condition or behavior, it must finst of all be a component of a larger system. This system has otructure in the form of relations among its components. A pattern is a property of the system, namely, the property of a subset of these stuctural relations holding. A norm is a pattern which is established either by conventional stipulation or by statistical regularity. An entity is behaving in a normative fashion if it is a component of a system and instantiates a norm within that system. The word "operate" given above illustrates this. When we say that an eogine is operating, we have in mind a larger system, the device the engine drives, to which the engine may bear various possible relations. A subset of these relations is stipulated to be the norm-the way it is supposed to work. We say it is operating when it is instantiating this norm.

\section{Conclusion}

The research we have been engaged in has forced us to explicate a complex set of commonsense concepts. Since we have done it in as general a fashion as possible, we may expect that it will be possible to axiomatize a large number of other areas, including areas unrelated to mechanical devices, building on this foundation. The very fact that we have been able to characterize words as diverse as "range", "immediately", "brittle", "operate" and "wear" shows the promise of this approach.

\section{Acknowledgements}

The research reported bere was funded by the Delense $A$ d. vanced Research Projects Agency under Office of Naval Research contract N00014-85-C-0013. It builds on work supported by NIH Grant LM03611 from the National Library of Medicine, by Grant IST-8209346 from the National Science Foundation, and by a gift from the Systems Development Foundation.

\section{References}

[1] Allen, James F., and Benry A. Kautz. 1985. "A model of naive temporal reasoning." Formal Theoried of the Commonsense World, ed. by Jerry R. Hobbs and Robert C. Moore, Ablex Publishing Corp., 251-268.
[2] Croft, William. 1986. Calegories and Relations in Syn. lax: The Clarse-Level Orpanization of Information. Ph.D. dissertation, Department of Linguistics, Stanford University.

[3] Davies, Todd R., and Stuart J. Russell. 1986. "A logical approach to reasoning by analogy." Submitted to the AAAI-86 Fifth National Conference on Artificial Intelligence, Pbiladelphia, Pennsylvania.

14] Davis, Emest. 1984. "Shape and Function of Solid Objects: Some Examples." Computer Science Technical Report 137, New York University. October 1984.

[5] Hager, Greg. 1985. "Naive physics of materials: A recon mission." In Commonsense Summer: Final Report, Report No. CSLI-85-35, Center for the Study of Language and Information, Stanford University.

[6] Hayes, Patrick J. 1979. "Naive physics manifesto." Ex. pert Systems in the Micro-electronic Age, ed. by Donald Michie, Edinburgh University Press, pp. 242-270.

[7] Herskovits, Annette. 1982. Space and the Preposilions in English: Regularities and Irregularilies in a Complez Domain. Ph.D. dissertation, Department of Linguistics, Stanford University.

[8] Hilbert, David. 1902. The Foundations of Geometry. The Open Court Publishing Company.

[9] Hobbs, Jerry R. 1974. "A Model for Natural Language Semantics, Part I: The Model." Research Report \#36, Department of Computer Science, Yale University. October 1974.

[10] Hobbs, Jerry R. 1985a. "Ontological promiscuity." Proceedings, esrd Annval Meeting of the Association for Computational Lingristics, pp. 61-69.

[11] Hobbs, Jerry R. 1985b. "Granularity:" Proceedings of the Ninth International Joint Conference on Artificial Intelligence, Los Angeles, California, August 1985, 432. 435.

[12] Hobbs, Jerry R. and Robert C. Moore, eds. 1985. Formal Theories of the Commonsense W'orld, Ablex Publishing Corp.

[13] Hobbs, Jerty R et al. 1985. Commonsense Summer: Final Report, Report No. CSLI-85-35, Center for the Study of Language and Information, Stanford University.

[14] Katz, Jerrold J. and Jerry A. Fodor. 1963. "The struture of a semantic tbeory." Language, Vol. 39 (AprilJune 1963), 170-210. 
[15] Lakoff, G. 1972. "Linguistics and natural logic". Semantica of Nalural Language, ed. by Donald Davidson and Gilbert Harman, 545-665.

[16] McDermott, Drew. 1985. "Reasoning about plans." Formal Thearies of the Commonsense Wortd, ed. by Jerry R. Hobbs and Robert C. Moore, Ablex Publishing Corp., 269-318.

[17] Miller, George A. and Philip N. Johnson-Laird. 1976. Langage and Pereeplion, Belknap Press.

[18] Rieger, Charles J. 1974. "Conceptual memory: A theory and computer program for processing and meaning content of natural language utterances." Stanfond AIM233, Department of Computer Science, Stanford University.

[19] Schank, Roger. 1975. Conceplual Information Processing. Elsevier Publishing Company.

[20] Shobam, Yoav. 1985. "Naive kinematics: Two aspects of shape." ln Commonsense Summer: Final Report, Re port No. CSLI-85-35, Center for the Study of Language and Information, Stanford University.

[21] Stickel, M.E. 1982. “A nonclausal connection-graph resolution theorem-proving program." Proceedings of the AAAl-82 National Conference on Arlificial Intelligence, Pittsburgh, Pennsyliania, 229-233.

[22] Talmy, Leonard. 1983. "How language structures space." Spatial Orientation: Theory, Researeh, and Application, ed. by Herbert Pick and Linda Acredolo, Plenum Press.

[23] Talmy, Leonard. 1985. "Force dynamics in language and thought." Proceedings from the Parasession on Causatives and Agentivity, 21 st Regional Meeting, Chicago Linguistic Society, ed. by William B. Eilfort, Paul D. Kroeber, and Karen L. Peterson.

[24] Zabn, C. T., and R. Z. Roskies. 1972. "Fourier descriptors for plane closed curves." IEEE Transactions on Computers, Vol. C-21, No. 3. 269-281. March 1972. 